Bulletin of the Section of Logic

Volume 47/3 (2018), pp. 187-200

http://dx.doi.org/10.18778/0138-0680.47.3.04

Rafał Gruszczyński and Andrzej Pietruszczak

\title{
A COMPARISON OF TWO SYSTEMS OF POINT-FREE TOPOLOGY
}

\begin{abstract}
This is a spin-off paper to [3, 4] in which we carried out an extensive analysis of Andrzej Grzegorczyk's point-free topology from [5]. In [1] Loredana Biacino and Giangiacomo Gerla presented an axiomatization which was inspired by the Grzegorczyk's system, and which is its variation. Our aim is to compare the two approaches and show that they are slightly different. Except for pointing to dissimilarities, we also demonstrate that the theories coincide (in the sense that their axioms are satisfied in the same class of structures) in presence of axiom stipulating non-existence of atoms.

Keywords: point-free topology, region-based topology, foundations of topology, mereology, mereological structures, separation structures, connection structures, Grzegorczyk structures, Biacino-Gerla structures.
\end{abstract}

\section{Mereology}

The underlying theory of point-free topologies presented in the sequel is mereology, the theory of wholes and their parts. For the sake of this paper we will assume mereology in its strongest complete form.

The only primitive notions are individual notion of region and relational notion of being part of. The set of regions will be denoted by ' $R$ ' and parthood by means of 'ᄃ’. We standardly assume that $\sqsubseteq$ is a partial order: it is reflexive, antisymmetric and transitive, i.e., we have: 


$$
\begin{gathered}
\forall_{x \in R} x \sqsubseteq x, \\
\forall_{x, y \in R}(x \sqsubseteq y \wedge y \sqsubseteq x \Longrightarrow x=y), \\
\forall_{x, y, z \in R}(x \sqsubseteq y \wedge y \sqsubseteq z \Longrightarrow x \sqsubseteq z) .
\end{gathered}
$$

By means of $\sqsubseteq$ we introduce three auxiliary relations: of being proper part, of overlapping and of being exterior to, respectively:

$$
\begin{aligned}
& \forall_{x, y \in R}(x \sqsubset y: \Longleftrightarrow x \sqsubseteq y \wedge x \neq y), \\
& \forall_{x, y \in R}\left(x \circ y: \Longleftrightarrow \exists_{z \in R}(z \sqsubseteq x \wedge z \sqsubseteq y)\right), \\
& \forall_{x, y \in R}(x \backslash y: \Longleftrightarrow \neg x \bigcirc y) .
\end{aligned}
$$

We assume that $\sqsubseteq$ is separative:

$$
\forall_{x, y \in R}\left(x \nsubseteq y \Longrightarrow \exists_{z \in R}(z \sqsubseteq x \wedge z 2 y)\right)
$$

and that it satisfies completeness axiom for mereological sums:

$$
\forall_{S \in \mathcal{P}^{+}(R)} \exists_{x \in R} x \text { sum } S,
$$

where sum is the standard mereological sum relation included in $R \times \mathcal{P}(R)$ and defined via:

$$
x \text { sum } S: \Longleftrightarrow \forall_{z \in S} z \sqsubseteq x \wedge \forall_{y \in R}\left(y \sqsubseteq x \Longrightarrow \exists_{z \in S} z \circ y\right) . \quad \text { (dfsum) }
$$

Note that, by $\left(\mathrm{r}_{\sqsubseteq}\right)$, we have:

$$
\forall_{S \in \mathcal{P}(S)} \forall_{x \in R}(x \text { sum } S \Longrightarrow S \neq \emptyset) .
$$

Moreover, we obtain:

$$
\forall_{S \in \mathcal{P}(S)} \forall_{x, y \in R}(x \operatorname{sum} S \wedge y \text { sum } S \Longrightarrow x=y) .
$$

We define MS to be the class of mereological structures, i.e., the class of separative partial orders which satisfy ( $\exists$ sum).

By means of the sum relation we define the standard mereological operations, i.e. the operation of mereological sum:

$$
x \sqcup y:=(\mathrm{l} z) z \operatorname{sum}\{x, y\},
$$

the partial operation of mereological product:

$$
x \circ y \Longrightarrow x \sqcap y:=(\mathrm{l} z) z \text { sum }\{u \in R \mid u \sqsubseteq y \wedge u \sqsubseteq x\},
$$


the partial operation of mereological complement:

$$
x \neq 1 \Longrightarrow-x:=(\mathrm{l} z) z \text { sum }\{u \in R \mid u\{x\},
$$

and the partial operation of relative complement:

$$
x \nsubseteq y \Longrightarrow x-y:=(\mathrm{l} z) z \text { sum }\{u \in R \mid u \sqsubseteq x \wedge u 2 y\} .
$$

In case $y \sqsubset x$, irreflexivity of proper part relation and antisymmetry of parthood entail that $x \nsubseteq y$, so there exists $x-y$.

Let $\mathrm{At}_{\mathfrak{M}}$ be the set of atoms of a mereological structure $\mathfrak{M}=\langle R, \sqsubseteq\rangle$, that is of all elements which are minimal with respect to part of relation $\sqsubset$. Let us remind that $\mathfrak{M}$ is atomic iff every its region has an atom, i.e., the following condition $\forall_{x \in R} \exists_{a \in \mathrm{At}_{\mathfrak{M}}} a \sqsubseteq x$ holds. Moreover, $\mathfrak{M}$ is atomless iff there are no atoms in $\mathfrak{R}$. Obviously, by definition, $A t_{\mathfrak{M}}=\emptyset$ iff the following condition holds:

$$
\forall x \in R \exists y \in R \quad y \sqsubset x .
$$

In the sequel we will refer to consequences of the axioms and definitions, but we omit their proofs for obvious reasons. The reader interested in details is requested to consult [6].

Convention. If $\boldsymbol{K}$ is a class of structures and $\varphi_{1}, \ldots, \varphi_{n}$ are conditions formulated in their language, then $\boldsymbol{K}+\varphi_{1}+\cdots+\varphi_{n}$ is a subclass of $\boldsymbol{K}$ which consists of all structures from $\boldsymbol{K}$ which additionally satisfy all $\varphi_{1}, \ldots, \varphi_{n}$.

\section{Quasi-separation relation}

\subsection{Separation relation}

Quasi-separation structures are obtained from mereological structures by adding a binary separation relation $)($ on regions with the following three postulates:

$$
\begin{gathered}
\forall_{x, y \in R}(x)(y \Longrightarrow x \nsubseteq y), \\
\forall_{x, y \in R}(x)(y \Longrightarrow y)(x), \\
\forall_{x, y \in R}\left(x \sqsubseteq y \Longrightarrow \forall_{z \in R}(z)(y \Rightarrow z)(x)\right) .
\end{gathered}
$$

From the axioms it follows that $x$ is irreflexive and that it is included in ?:

$$
\begin{gathered}
\left.\forall_{x \in R} \neg x\right)(x, \\
\forall_{x, y \in R}(x)(y \Longrightarrow x(y) .
\end{gathered}
$$


We also obtain that:

$$
\forall_{x, y, z \in R}(z)(x \sqcup y \Longrightarrow z)(x \wedge z)(y) .
$$

Let qSep $:=\mathbf{M S}+(\mathrm{S} 1)+(\mathrm{S} 2)+(\mathrm{S} 3)$ be the class of all quasi-separation structures. $^{1}$

We have the following equivalent axiomatizations of this class:

Proposition 2.1. For any relational structure $\mathfrak{R}=\langle R, \sqsubseteq)(\rangle$, such that $\langle R, \sqsubseteq\rangle$ belongs to $\mathbf{M S}$ and $)(\subseteq R \times R$ the following conditions are equivalent:

(a) $\mathfrak{R} \in \mathbf{q S e p}$.

(b) $\mathfrak{R}$ satisfies conditions $\left(\operatorname{irr}_{)}\right)$, (S2), and (S3).

(c) $\mathfrak{R}$ satisfies conditions $\left(\mathrm{I}_{)}^{2}\right)$, (S2), and (S3).

\subsection{Connection relation}

In any quasi-separation structure $\mathfrak{R}=\langle R, \sqsubseteq)(\rangle$, we also introduce an auxiliary binary connection (or contact) relation $\mathrm{C}$ in $R$ :

$$
\forall x, y \in R(x \mathrm{C} y: \Longleftrightarrow \neg x)(y),
$$

By (dfC) and, respectively, (S1)-(S3), $\left(\operatorname{irr}_{)}\right),\left(\mathrm{I}_{)}^{2}\right),(2.1)$ the following conditions hold:

$$
\begin{gathered}
\forall_{x, y \in R}(x \sqsubseteq y \Longrightarrow x \mathrm{C} y), \\
\forall_{x, y \in R}(x \mathrm{C} y \Longrightarrow y \mathrm{C} x), \\
\forall_{x, y \in R}\left(x \sqsubseteq y \Longrightarrow \forall_{z \in R}(z \mathrm{C} x \Rightarrow z \mathrm{C} y)\right), \\
\forall_{x \in R} x \mathrm{C} x, \\
\forall_{x, y \in R}(x \circ y \Longrightarrow x \mathrm{C} y), \\
\forall_{x, y, z \in R}(z \mathrm{C} x \vee z \mathrm{C} y \Longrightarrow z \mathrm{C} x \sqcup y) .
\end{gathered}
$$

Every relational structure $\langle R, \sqsubseteq, \mathrm{C}\rangle$ satisfying all axioms for mereological structures plus (C1)-(C3) is called a quasi-connection structure.

Proposition 2.2. Any quasi-separation structure $\langle R, \sqsubseteq, x\rangle$ is definitionally equivalent to the quasi-connection structure $\langle R, \sqsubseteq, \mathrm{C}\rangle$ via $(\mathrm{df} \mathrm{C})$ and the following formula:

$$
\forall x, y \in R(x)(y \Longleftrightarrow \neg x \text { C } y) .
$$

\footnotetext{
${ }^{1}$ The symbols 'qSep', 'Sep' and 'G' (see further) are used in [3, 4], but there their meaning is different, since in the aforementioned papers we take into account structures which are not complete. Thus the reader who is familiar with the two papers is asked to bear in mind that all structures analyzed in this paper are complete.
} 


\subsection{Non-tangential inclusion of regions}

Yet another relation defined by means of the separation is that of nontangential inclusion $\ll$ :

$$
\forall_{x, y \in R}\left(x \ll y: \Longleftrightarrow \forall_{z \in R}(y \nmid z \Rightarrow x)(z)\right) .
$$

The following facts were proven, among others, in [1, 3]:

$$
\begin{gathered}
\forall_{x, y \in R}(x \ll y \Longrightarrow x \sqsubseteq y), \\
\forall_{x, y \in R}\left(\forall_{u \in R} u \sqsubseteq y \Longrightarrow x \ll y\right), \\
\forall_{x, y \in R}\left(x \ll y \Longrightarrow \forall_{u \in R} u \sqsubseteq y \vee \exists_{z \in R}(z\{y \wedge z)(x)),\right. \\
\forall_{x, y \in R}(x \ll y \wedge y \ll x \Longrightarrow x=y), \\
\forall_{x, y, z \in R}(x \ll y \wedge y \sqsubseteq z \Longrightarrow x \ll z), \\
\forall_{x, y, z \in R}(x \sqsubseteq y \wedge y \ll z \Longrightarrow x \ll z), \\
\forall_{x, y, z \in R}(x \ll y \wedge y \ll z \Longrightarrow x \ll z) .
\end{gathered}
$$

Conditions (2.2) and (2.3) entail that:

$$
\begin{gathered}
\forall_{x \in R} x \ll 1, \\
\forall_{x, y \in R}\left(x \ll y \Longrightarrow y=1 \vee \exists_{z \in R}(z 2 y \wedge z)(x)\right) .
\end{gathered}
$$

Therefore we have the following characterization of the relation $\ll$ :

$$
\forall_{x, y \in R}(x \ll y \Longleftrightarrow y=1 \vee(y \neq 1 \wedge x)(-y)) .
$$

Proposition 2.3. For any quasi-separation structure:

1. $\sqsubseteq$ is included in $\ll$ iff $\left.\forall_{y \in R \backslash\{1\}} y\right)(-y$.

2. $x=\emptyset \quad$ iff $\quad \ll \subseteq R \times\{1\} \quad$ iff $\quad \ll=R \times\{1\}$.

\section{Separation structures}

A quasi-separation structure $\langle R, \sqsubseteq, x\rangle$ is turned into a separation structure by requiring that it satisfies both implications converse to (S3) and (2.1):

$$
\begin{gathered}
\forall_{x, y, z \in R}(z)(x \wedge z)(y \Longrightarrow z)(x \sqcup y) \\
\forall_{x, y \in R}\left(\forall_{z \in R}(z)(y \Rightarrow z)(x) \Longrightarrow x \sqsubseteq y\right) .
\end{gathered}
$$

We define Sep $:=\mathbf{q S e p}+(\mathrm{S} 4)+(\mathrm{S} 5)$. As it was observed in [3], in all structures from Sep, by (S3) and (S5), the relation $\sqsubseteq$ is definable by )x, i.e., we have: 


$$
\forall_{x, y \in R}\left(x \sqsubseteq y \Longleftrightarrow \forall_{z \in R}(z)(y \Rightarrow z)(x)\right) .
$$

Again, in [3] the following facts about separation structures were established:

$$
\begin{gathered}
\forall_{x, y, z \in R}(z \mathrm{C} x \sqcup y \Longrightarrow z \mathrm{C} x \vee z \mathrm{C} y), \\
\forall_{x, y \in R}\left(\forall_{z \in R}(z \mathrm{C} x \Rightarrow z \mathrm{C} y) \Longrightarrow x \sqsubseteq y\right), \\
\forall_{x, y \in R}\left(x \sqsubseteq y \Longleftrightarrow \forall_{z \in R}(z \mathrm{C} x \Rightarrow z \mathrm{C} y)\right), \\
\forall_{x, y, z \in R}(z)(x \sqcup y \Longleftrightarrow z)(x \wedge z)(y), \\
\forall_{x, y, z \in R}(z \mathrm{C} x \sqcup y \Longleftrightarrow z \mathrm{C} x \vee z \mathrm{C} y) .
\end{gathered}
$$

All quasi-connection structures that satisfy (C4) and (C5) are called connection structures (but see Remark 1.1 in [3]).

«Standard» quasi-separation (resp. separation) structures are obtained from topological spaces by means of the following method. For any topological space $\mathcal{T}=\langle S, \mathscr{O}\rangle$, the pair $\left\langle\mathrm{r} \mathscr{O}^{+}, \subseteq\right\rangle$ is a mereological structure (see for example Lemma 2.9 in [3]). The separation relation ][ in $\mathrm{r} \mathscr{O}^{+}$is introduced by:

$$
U][V: \Longleftrightarrow \mathrm{Cl} U \cap \mathrm{Cl} V=\emptyset .
$$

The connection and non-tangential inclusion are characterized in this kind of structures by the following two conditions (being consequences of (df][) and the axioms):

$$
\begin{aligned}
U \subset V & \Longleftrightarrow \mathrm{Cl} U \cap \mathrm{Cl} V=\emptyset, \\
U \ll V & \Longleftrightarrow \mathrm{Cl} U \subseteq V .
\end{aligned}
$$

\section{Grzegorczyk structures}

\subsection{Representatives of points}

Let $\mathfrak{R}=\langle R, \sqsubseteq, x\rangle$ be an arbitrary quasi-separation structure. After Grzegorczyk [5], by a representative of a point (or a pre-point) of $\mathfrak{R}$ we mean any non-empty subset $X$ of $R$ satisfying the following three conditions: ${ }^{2}$

$$
\begin{gathered}
\forall_{u, v \in X}(u=v \vee u \ll v \vee v \ll u), \\
\forall_{u \in X} \exists_{v \in X} v \ll u, \\
\forall_{x, y \in R}\left(\forall_{u \in X}(u \circ x \wedge u \circ y) \Longrightarrow x \subset y\right) .
\end{gathered}
$$

\footnotetext{
${ }^{2}$ The reader interested in geometrical intuitions behind pre-points (and points) is asked to consult [2].
} 
Let $\mathbf{Q}_{\mathfrak{R}}$ be the family of all pre-points of $\mathfrak{R}$, i.e.:

$$
Q \in \mathbf{Q}_{\mathfrak{R}}: \Longleftrightarrow \emptyset \neq Q \subseteq R \text { and } Q \text { satisfies }(\mathrm{r} 1)-(\mathrm{r} 3) .
$$

In the light of $(\mathrm{r} 1),\left(\mathrm{I}_{\ll}\right)$ and the fact that $x \sqsubseteq y \Longleftrightarrow x \sqcap y=x$, for all $Q \in \mathbf{Q}_{\Re}$ and $u_{1}, u_{2} \in Q$ we have: either $u_{1} \sqcap u_{2}=u_{1}$ or $u_{1} \sqcap u_{2}=u_{2}$. So we obtain that any $Q \in \mathbf{Q}_{\mathfrak{R}}$ generates the filter $\mathrm{F}_{Q}:=\left\{x \in R \mid \exists_{u \in Q} u \sqsubseteq x\right\}$. By a point of $\mathfrak{R}$ we mean any filter generated by a pre-point of $\mathfrak{R}$ :

$$
\mathbf{P t}_{\mathfrak{R}}:=\left\{X \in \mathcal{P}^{+}(R) \mid \exists_{Q \in \mathbf{Q}_{\mathfrak{R}}} X=\mathrm{F}_{Q}\right\} .
$$

Elements of the set $\mathbf{P} \mathbf{t}_{\mathfrak{R}}$ will be denoted by small Gothic letters ' $\mathfrak{p}$ ' and ' $\mathfrak{q}$ '.

\subsection{Grzegorczyk's axiom}

The Grzegorczyk's axiom from [5] says that in a quasi-separation structure $\mathfrak{R}=\langle R, \sqsubseteq, x\rangle$ for any connected regions $x, y \in R$ there exists a pre-point $Q$ such that:

(g1) either $x 2 y$ or there is $z \in Q$ such that $z \sqsubseteq x \sqcap y$,

(g2) for any $z \in Q$ we have $z \circ x$ and $z \circ y$.

Formally: ${ }^{3}$

$\forall_{x, y \in R}\left(x \subset y \Longrightarrow \exists_{Q \in \mathbf{Q}_{\Re}}\left(\left(x \nmid y \vee \exists_{z \in Q} z \sqsubseteq x \sqcap y\right) \wedge \forall_{z \in Q}(z \circ x \wedge z \circ y)\right)\right)$.

By a Grzegorczyk structure (or G-structure) we mean any quasi-separation structure satisfying $(G)$. Let $\mathbf{G}:=\mathbf{q S e p}+(\mathrm{G})$ be the class of all $\mathrm{G}$ structures. In light of $(\mathrm{G}),\left(\mathrm{I}_{\circ}^{\mathrm{C}}\right)$ and the mereological axioms, reflexivity of $\bigcirc$ and $C$ entail that: $\mathbf{Q}_{\mathfrak{R}} \neq \emptyset$ and so $\mathbf{P t}_{\mathfrak{R}} \neq \emptyset$.

Now notice that:

Proposition 4.1 ([3, Proposition 6.1]). The axiom (G) can be replaced with the conjunction of the following two simpler conditions:

$$
\begin{gathered}
\forall_{x, y \in R}\left(x \circ y \Longrightarrow \exists_{Q \in \mathbf{Q}_{\mathfrak{R}}} \exists_{z \in Q} z \sqsubseteq x \sqcap y\right), \\
\forall_{x, y \in R}\left(x \subset y \wedge x 2 y \Longrightarrow \exists_{Q \in \mathbf{Q}_{\mathfrak{R}}} \forall_{z \in Q}(z \circ x \wedge z \circ y)\right) .
\end{gathered}
$$

${ }^{3}$ Strictly speaking, the axiom introduced by us is different from that presented in [5]. However, in [4] we demonstrated that both axiomatizations are equivalent to each other. 
From axioms of G-structures we obtain:

$$
\begin{gathered}
\forall_{x \in R} \exists_{Q \in \mathbf{Q}_{\mathfrak{R}}} \exists_{z \in Q} z \sqsubseteq x \text { and } \quad \forall_{x \in R} \exists_{\mathfrak{p} \in \mathbf{P t}_{\mathfrak{M}}} x \in \mathfrak{p}, \\
\forall_{x, y \in R}\left(x \mathrm{C} y \Longrightarrow \exists_{\mathfrak{p} \in \mathbf{P t}_{\mathfrak{R}}} \forall_{z \in \mathfrak{p}}(z \circ x \wedge z \bigcirc y)\right)
\end{gathered}
$$

Proof: $A d$ (4.1): Since $x \bigcirc x$, by $\left(\mathrm{G}_{\circ}\right)$, there is a pre-point $Q$ such that for some $z \in Q$ we have $z \sqsubseteq x$. Hence $\mathrm{F}_{Q}$ is a point which contains $x$.

Ad (4.2): Assume that $x \mathrm{C} y$. Then, by $(\mathrm{G})$, there is a $Q \in \mathbf{Q}_{\mathfrak{R}}$ such that every region of $Q$ overlaps $x$ and $y$. Thus, also every region of the point $\mathrm{F}_{Q}$ overlaps $x$ and $y$.

In all G-structures the following two facts hold.

Proposition 4.2. Every region of a G-structure has at least one nontangential part. Formally: $\forall_{x \in R} \exists_{z \in R} z \ll x$.

Proof: By (4.1), for any $x \in R$ there are $Q \in \mathbf{Q}_{\mathfrak{R}}$ and $y \in Q$ such that $y \sqsubseteq x$. But $Q$ has the property (r2). Hence for some $z \in Q$ we have $z \ll y$; and so $z \ll x$, by (2.4).

Proposition 4.3. Every region is the sum of its non-tangential parts. Formally, for any $x \in R$ we have: $x$ sum $\{z \in R \mid z \ll x\}$.

Proof: Let $x \in R$ and $S:=\{z \in R \mid z \ll x\}$. Firstly, in light of ( $\left.\mathrm{I}_{\ll}^{\sqsubset}\right)$, for any $z \in S$ we have $z \sqsubseteq x$. Secondly, suppose that $y \sqsubseteq x$. Then, by Proposition 4.2, for some $z \in R$ we have $z \ll y$. So $z \ll x$, by (2.4), i.e., $z \in S$. Moreover, $z \sqsubseteq y$, by $\left(\mathrm{I}_{\ll}^{\sqsubset}\right)$. So $z \bigcirc y$, by $\left(\mathrm{r}_{\sqsubseteq}\right)$. Thus, $x$ sum $S$.

A non-trivial fact about $G$-structures is contained in the following:

THEOREM 4.4 ([4]). All G-structures are separation structures.

\subsection{Atoms of G-structures}

As it will turn out, the main difference between systems from [5] and [1] lies in the fact that $G$-structures, unlike Biacino-Gerla structures from Section 5 , admit atoms ${ }^{4}$ and may be finite.

Proposition 4.5 ([4]). For any G-structure $\mathfrak{R}$, for any $a \in \mathrm{At}_{\mathfrak{R}}: a \ll a$ and $\{a\} \in \mathbf{Q}_{\mathfrak{R}}$.

Proof: Let $a \in \mathrm{At}_{\mathfrak{R}}$. From Proposition 4.2 for some $x \in R$ we have $x \ll a$. Moreover, by ( $\left.\mathrm{I}_{\ll}^{\sqsubset}\right)$, we have $x=a$. So $a \ll a$.

\footnotetext{
${ }^{4}$ For any structure $\mathfrak{R}=\langle R, \sqsubseteq)(\rangle$, such that $\mathfrak{M}:=\langle R, \sqsubseteq\rangle$ is a mereological structure, atoms of $\mathfrak{R}$ are the atoms of $\mathfrak{M}$; i.e., we put $A t_{\mathfrak{R}}:=A t_{\mathfrak{M}}$. Moreover, we say that $\mathfrak{R}$ is atomic (resp. atomless) iff $\mathfrak{M}$ is atomic (resp. atomless).
} 
For $\{a\}$ condition (r1) is trivial and (r2) follows from the previous paragraph. For (r3), if $x, y \in R$ and $a \bigcirc x$ and $a \bigcirc y$, then $a \sqsubseteq x$ and $a \sqsubseteq y$. Hence $x \bigcirc y$; and therefore $x \subset y$, by $\left(\mathrm{I}_{\circ}^{\mathrm{C}}\right)$.

Proposition 4.6 ([3, Proposition 6.8]). Every atomic separation structure $\mathfrak{R}=\langle R, \sqsubseteq, 2\rangle$ is a $G$-structure.

Proof: It is routine to verify that $\mathfrak{R}$ is a quasi separation structure. Let $x, y \in R$ be such that $x \circ y$. Then exist both $x \sqcap y$ and $a \in \mathrm{At}_{\mathfrak{R}}$ such that $a \sqsubseteq x \sqcap y$. But $\{a\} \in \mathbf{Q}_{\mathfrak{R}}$, by Proposition 4.5. Further, since $\mathrm{C}=\mathrm{O}$, the second conjunct from the consequent of $(\mathrm{G})$ holds as well.

In the final section we will make use the following result (where we define $\left.\mathrm{F}_{x}:=\{y \in R \mid x \sqsubseteq y\}\right)$ :

Proposition 4.7 ([4, Proposition 12.7]). For any G-structure $\mathfrak{R}$, for any $x \in R: \mathrm{F}_{x} \in \mathbf{P t}_{\mathfrak{R}}$ iff $x \in \mathrm{At}_{\mathfrak{R}}$.

There are finite mereological structures, therefore there exist finite Grzegorczyk structures. The simplest example being the trivial structure $\langle\{1\}, \sqsubseteq, \emptyset\rangle$ which has only one pre-point $\{1\}$, being also the only point of this structure.

The following fact reveals an interesting dependency between the separation relation and the situation in which $\{1\}$ is among pre-points:

Proposition 4.8. For any G-structure $\mathfrak{R}$ :

1. If $\{1\} \in \mathbf{Q}_{\mathfrak{R}}$, then $)(=\emptyset$.

2. If $x=\emptyset$, then $\mathbf{Q}_{\mathfrak{R}}=\{\{1\}\}$.

3. The trivial structure is the unique structure in which $\{1\}$ is a pre-point.

Proof: Ad 1. Suppose that $\{1\} \in \mathbf{Q}_{\mathfrak{R}}$. Since for all $x, y \in R$ we have $x \circ 1 \bigcirc y$, so $x \mathrm{C} y$, by (r3). That is $\mathrm{C}=R \times R$; so $)(=\emptyset$.

$A d 2$. Let $)(=\emptyset$. Then $\mathrm{C}=R \times R$ and $\ll=R \times\{1\}$, by Proposition 2.3(2). First, notice that $\{1\} \in \mathbf{Q}_{\mathfrak{R}}$. Indeed, (r1) is trivial; (r2) by $1 \ll 1 ;(\mathrm{r} 3)$ by $\mathrm{C}=R \times R$. Second, suppose that $X \in \mathbf{Q}_{\mathfrak{R}}$ and $X \neq\{1\}$. Then there is an $x \in X$ such that $x \neq 1$. By (r2) there is a $y \in X$ such that $y \ll x$. But then $x=1$; a contradiction.

Ad 3. Suppose that $\mathfrak{R}$ is non-trivial and $\{1\} \in \mathbf{Q}_{\mathfrak{R}}$. Then for some $x \in R$ we have $x \sqsubset 1$ and, by (4.1), there are $Q \in \mathbf{Q}_{\mathfrak{R}}$ and $y \in R$ such that $y \in Q$ and $y \sqsubseteq x$. Since $y \neq 1$, we obtain a contradiction.

Now we consider the non-trivial $G$-structure which is built upon the three-element mereological structure with $x:=$ : 


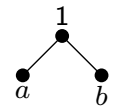

where $a \sqsubset 1, b \sqsubset 1, a\{b$ and $a)(b$. So it has four pre-points: $\{a\},\{b\}$, $\{a, 1\}$ and $\{b, 1\}$; and it has two points: $\{a, 1\}$ and $\{b, 1\}$. Interestingly, the trivial G-structure and the above three-element G-structure are the only $G$-structures for which $\mathbf{P t}_{\mathfrak{R}} \subseteq \mathbf{Q}_{\mathfrak{R}}$, and for others G-structures we always have $\mathbf{P t}_{\mathfrak{R}} \cap \mathbf{Q}_{\mathfrak{R}}=\emptyset$. This follows from the following facts: (a) all pre-points are chains and (b) if a G-structure has more than three elements then none of its points is a chain.

For (b): In the case of any infinite structure, every point $\mathfrak{p}$ must be infinite, so there are $x, y \in \mathfrak{p}$ such that $x \sqsubset y \sqsubset 1$. So $x \sqcup-y$ belongs to $\mathfrak{p}$ but it is incomparable to $y$ with respect to $\sqsubseteq$.

Now suppose that a G-structure has more than two atoms and $a$ is an arbitrary atom. Then there are two atoms $b$ and $c$ such that $a \neq b \neq c \neq a$ and we have: $a \sqsubset a \sqcup b \sqsubset 1, a \sqsubset a \sqcup c \sqsubset 1$ and $a=(a \sqcup b) \sqcap(a \sqcup c)$. So $a \sqcup b \in \mathrm{F}_{a}$ and $a \sqcup c \in \mathrm{F}_{a}$. However, $a \sqcup b$ and $a \sqcup c$ are incomparable with respect to $\sqsubseteq$. Thus, the filter $\mathrm{F}_{a}$ is not a chain. In consequence, in the case when $\mathfrak{R}$ is finite and has at least three atoms we use the following:

Proposition 4.9 ([4, Proposition 12.9]). If a G-structure $\mathfrak{R}$ is finite, then $\mathbf{P t}_{\mathfrak{R}}=\left\{\mathrm{F}_{a} \mid a \in \mathrm{At}_{\mathfrak{R}}\right\}$.

\section{Biacino-Gerla structures}

Let $\mathfrak{R}=\langle R, \sqsubseteq, \boldsymbol{x}\rangle$ be an arbitrary separation structure. In the BiacinoGerla's system from [1] the notion of representative of a point is defined as a set $Q$ of regions such that (see [1, p. 434]):

(A) $Q$ does not have minimum and any its distinct elements are comparable with respect to $\ll$,

(B) if regions $x$ and $y$ overlap every region $u \in Q$, then $x \mathrm{C} y$.

Obviously, condition (B) coincides with condition (r3) in Grzegorczyk's definition of pre-point. Furthermore, condition (A) is equivalent to the pair of conditions: (r1) plus the following stronger version of condition (r2):

$$
\forall u \in R \exists_{v \in R}(v \ll u \wedge v \neq u) .
$$

Therefore, we define the set $\mathbf{Q}_{\mathfrak{R}}^{\star}$ of all pre-points in the sense of Biacino's and Gerla's as the set of all non-empty sets of regions which satisfy (r1), 
$\left(\mathrm{r} 2^{\prime}\right)$ and (r3). Points are defined as before, as filters generated by representatives of points. Let $\mathbf{P t}_{\mathfrak{R}}^{\star}$ be the set of points in the sense of the Italian mathematicians'.

Due to the above, $\left(\mathrm{I}_{\ll}\right)$ and irreflexivity of the proper part relation, we immediately have the following:

Proposition 5.1. 1. $\mathbf{Q}_{\mathfrak{R}}^{\star} \subseteq \mathbf{Q}_{\mathfrak{R}}$. So $\mathbf{P t}_{\mathfrak{R}}^{\star} \subseteq \mathbf{P t}_{\mathfrak{R}}$.

2. All pre-points of $\mathbf{Q}_{\mathfrak{R}}^{\star}$ are infinite. So all points from $\mathbf{P t}_{\mathfrak{R}}^{\star}$ are infinite.

There exist, however, separation structures (e.g. all finite ones) in which there are pre-points and points in the sense of Grzegorczyk's, but which have no pre-points as defined by Biacino-Gerla.

Two specific axioms accepted by Biacino-Gerla instead of $(G)$ are:

$$
\begin{gathered}
\forall_{x \in R} \exists_{\mathfrak{p} \in \mathbf{P t}_{\mathfrak{R}}^{\star}} x \in \mathfrak{p}, \\
\forall_{x, y \in R}\left(x \subset y \Longrightarrow \exists_{\mathfrak{p} \in \mathbf{P t}_{\mathfrak{\mathfrak { R }}}^{\star}} \forall_{z \in \mathfrak{p}}(z \circ x \wedge z \bigcirc y)\right) .
\end{gathered}
$$

Let us call any separation structure satisfying the above axioms BiacinoGerla structure (or BG-structure), and let BG be the class of all such structures.

For the sake of presentation, for $\mathfrak{p}$ being either a point in the sense of Grzegorczyk's or Biacino's and Gerla's, and $x$ a region, let us define:

$$
\forall_{x \in R} \forall_{\mathfrak{p} \in \mathbf{P t}_{\mathfrak{\Re}}^{\star}}\left(\mathfrak{p} \text { is adherent to } x: \Longleftrightarrow \forall_{y \in \mathfrak{p}} y \bigcirc x\right) .
$$

Clearly, Proposition 5.1(2) and axiom (BG1) entail that: Proposition 5.2. All BG-structures are infinite.

To prove the next proposition we will refer to Proposition 4.1. Proposition 5.3. BG $\subsetneq$ G.

Proof: We show that if $\mathfrak{R} \in \mathbf{B G}$, then $\mathfrak{R}$ satisfies both $\left(\mathrm{G}_{\circ}\right)$ and $\left(\mathrm{G}_{\mathfrak{}}\right)$. For $\left(\mathrm{G}_{\circ}\right)$ assume $x \circ y$. By (BG1), there is $\mathfrak{p} \in \mathbf{P t}_{\mathfrak{R}}^{\star}$ such that $x \sqcap y \in \mathfrak{p}$. Hence, by Proposition 5.1(1), there is a $Q \in \mathbf{Q}_{\mathfrak{R}}^{\star} \subseteq \mathbf{Q}_{\mathfrak{R}}$ such that $\mathfrak{p}=\mathrm{F}_{Q}$ and for some $z \in Q$ we have $z \sqsubseteq x \sqcap y$.

For $\left(\mathrm{G}_{\circ}\right)$ suppose that $x \mathrm{C} y$ and $x 2 y$. Then, by (BG2), there is a point $\mathfrak{p} \in \mathbf{P} \mathbf{t}_{\mathfrak{R}}^{\star}$ which is adherent to both $x$ and $y$. Hence there is a $Q \in \mathbf{Q}_{\mathfrak{R}}^{\star} \subseteq \mathbf{Q}_{\mathfrak{R}}$ such that $\mathfrak{p}=\mathrm{F}_{Q}$ and for any $z \in Q$ we have $z \bigcirc x$ and $z \bigcirc y$.

The inclusion of the classes in question is proper, since for example the three-element G-structure from p. 195 is not a BG-structure. Let us now prove, after Biacino and Gerla, the following: 
Proposition 5.4 ([1]). Any region of a BG-structure $\mathfrak{R}$ has non-tangential proper parts which are separated from one another. Formally:

$$
\forall_{x \in R} \exists_{u, v \in x}(u \ll x \wedge u \neq x \wedge v \ll x \wedge v \neq x \wedge u)(v) .
$$

Proof: Let $x \in R$. By (BG1), there is $\mathfrak{p} \in \mathbf{P t}_{\mathfrak{R}}^{\star}$ such that $x \in \mathfrak{p}$. Hence there is a $Q \in \mathbf{Q}_{\mathfrak{R}}^{\star}$ such that $\mathfrak{p}=\mathrm{F}_{Q}$ and for some $y \in Q$ we have $y \sqsubseteq x$. However, (r2 $\left.2^{\prime}\right)$ entails that for some $u \in Q$ we have: $u \ll y$ and $u \neq y$. From this, ( $\left.\mathrm{I}_{\ll}^{\mathrm{c}}\right)$ and axioms of mereology we get: $u \sqsubset y$ and so there is a region $y-u$. Moreover, by (2.4), ( $\left.\mathrm{I}_{\ll}^{\sqsubset}\right)$ and (antis ${ }_{\sqsubseteq}$ ), we have: $u \ll x$ and $u \neq x$. Again by (BG1), there is $\mathfrak{q} \in \mathbf{P t}_{\mathfrak{R}}^{\star}$ such that $y-u \in \mathfrak{q}$. Hence there is a $Q^{\prime} \in \mathbf{Q}_{\mathfrak{R}}^{\star}$ such that $\mathfrak{q}=\mathrm{F}_{Q^{\prime}}$ and for some $z \in Q$ we have $z \sqsubseteq y-u \sqsubseteq y \sqsubseteq x$. Moreover, again ( $\left.\mathrm{r} 2^{\prime}\right)$ entails that for some $v \in Q^{\prime}: v \ll z$ and $v \neq z$. So, by $\left(\mathrm{t}_{\sqsubseteq}\right),(2.4),\left(\mathrm{I}_{\ll}^{\sqsubset}\right)$ and (antis $\left.{ }_{\sqsubseteq}\right)$, we obtain: $v \ll x$ and $v \neq x$. Obviously, $y-u \neq 1$, so there exists $-(y-u)$. We have $-(y-u)=-(y \sqcap-u)$ and $z)(-(y \sqcap-u)$. But, by $(\mathrm{df} \ll)$, also $v)(-(y \sqcap-u)$. In the case $y=1$, we have: $-(y \sqcap-u)=--u=u$; and so $v)(u$. Otherwise, $-(y \sqcap-u)=-y \sqcup u$; and so $v)(-y \sqcup u$. This fact and (2.1) entail that $v)(u$.

Corollary 5.5 ([1]). Any region $x$ of a BG-structure $\mathfrak{R}$ has a nontangential proper part. Formally $\forall_{x \in R} \exists_{y \in R}(y \ll x \wedge y \neq x)$. So $\mathfrak{R}$ satisfies (atomless). ${ }^{5}$

Proof: Directly from Proposition 5.4. Hence, in the light of $\left(\mathrm{I}_{\ll}^{\sqsubset}\right)$ and (df $\sqsubset$ ), (atomless) holds.

Corollary 5.5 is a counterpart of Proposition 4.2. Now we get a counterpart of Proposition 4.3.

Proposition 5.6. Every region of a BG-structure is the mereological sum of all its non-tangential proper parts. Formally, for any $x \in R$ we have:

$$
x \text { sum }\{z \in R \mid z \ll x \wedge z \neq x\} .
$$

Proof: Let $x \in R$ and $S:=\{z \in R \mid z \ll x \wedge y \neq x\}$. Firstly, in light of $\left(\mathrm{I}_{\ll}^{\sqsubset}\right)$, for any $z \in S$ we have $z \sqsubseteq x$. Secondly, suppose that $y \sqsubseteq x$. Then, by Corollary 5.5, for some $z \in R$ we have $z \ll y$ and $z \neq y$. So $z \ll x$ and $z \neq x$, by $(2.4),\left(\mathrm{t}_{\sqsubseteq}\right)$ and $\left(\mathrm{I}_{\ll}^{\sqsubset}\right)$, i.e., $z \in S$. Moreover, $z \sqsubseteq y$, by $\left(\mathrm{I}_{\ll}\right)$. So $z \circ y$, by $\left(\mathrm{r}_{\sqsubseteq}\right)$. Thus, $x$ sum $S$.

For the class of $G$-structures, the above proposition does not hold. Indeed, if a G-structure has at least one atom $a$, then $\{x \in R \mid x \ll a \wedge x \neq$ $a\}=\emptyset$. Hence this set has no mereological sum, by (1.1).

\footnotetext{
${ }^{5}$ See footnote 4 .
} 
Finally, we prove that the class of BG-structures coincides with the class of atomless G-structures. To this end, we will apply the following lemma:

Lemma 5.7. For any atomless $G$-structure $\mathfrak{R}: \mathbf{Q}_{\mathfrak{R}}=\mathbf{Q}_{\mathfrak{R}}^{\star}$. In consequence $\mathbf{P t}_{\mathfrak{R}}=\mathbf{P t}_{\mathfrak{R}}^{\star}$.

Proof: Let $\mathfrak{R} \in \mathbf{G}+$ (atomless). In the light of Proposition 5.1(1), it suffices to show that $\mathbf{Q}_{\mathfrak{R}} \subseteq \mathbf{Q}_{\mathfrak{R}}^{\star}$, i.e., every pre-point of $\mathfrak{R}$ satisfies condition $\left(\mathrm{r} 2^{\prime}\right)$. Assume for a contradiction that a pre-point $Q$ of $\mathfrak{R}$ does not satisfy $\left(\mathrm{r} 2^{\prime}\right)$. Then for some $x \in Q$ the condition $\forall_{v \in Q}(v \ll x \Rightarrow v=x)$ holds. Hence, by (r2), $x \ll x$. So, by ( 11$)$, for any $y \in Q$ we have $x \ll y$. In consequence, in light of $\left(\mathrm{I}_{\kappa}^{\sqsubset}\right)$ and $\left(\mathrm{t}_{\sqsubseteq}\right)$, it must be that $\mathrm{F}_{Q}=\mathrm{F}_{x}$, i.e., $\mathrm{F}_{x} \in \mathbf{P t}_{\mathfrak{R}}$. From this and Proposition 4.7 we obtain that $x \in \mathrm{At}_{\mathfrak{R}}$; a contradiction.

TheOREM 5.8. $\mathbf{B G}=\mathbf{G}+$ (atomless).

Proof: From Proposition 5.3 and Corollary 5.5 we obtain the inclusion " $\subseteq$ ". Moreover, in the light of Lemma 5.7 and conditions (4.1) and (4.2), every atomless G-structure satisfies conditions (BG1) and (BG2), i.e., it belongs to BG.

Acknowledgements. Rafał Gruszczyński's work was supported by National Science Center, Poland, grant Applications of mereology in systems of point-free geometry, no. 2014/13/B/HS1/00766.

\section{References}

[1] L. Biacino and G. Gerla, Connection structures: Grzegorczyk's and Whitehead's definitions of point, Notre Dame Journal of Formal Logic 37 (3) (1996), pp. 431-439. http://dx.doi.org/10.1305/ndjll/1039886519.

[2] R. Gruszczyński and A. Pietruszczak, Space, points and mereology. On foundations of point-free Euclidean geometry. Logic and Logical Philosophy 18 (2) (2009), pp. 145-188. http://dx.doi.org/10.12775/LLP.2009.009.

[3] R. Gruszczyński and A. Pietruszczak, A study in Grzegorczyk point-free topology. Part I: Separation and Grzegorczyk structures, Studia Logica, Vol. 106 (2018), pp. 1197-1238. https://doi.org/10.1007/s11225-018-9786-8.

[4] R. Gruszczyński and A. Pietruszczak, A study in Grzegorczyk pointfree topology. Part II: Spaces of points, Studia Logica (2018). https://doi.org/10.1007/s11225-018-9822-8. 
[5] A. Grzegorczyk, Axiomatizability of geometry without points, Synthese 12 (2-3) (1960), pp. 228-235. DOI: http://dx.doi.org/10.1007/BF00485101.

[6] A. Pietruszczak, Metamereology, Nicolaus Copernicus University Scientific Publishing Hause, Toruń, 2018. DOI: http://dx.doi.org/10.12775/9751.

Department of Logic

Nicolaus Copernicus University in Toruń

ul. Moniuszki 16, 87-100 Toruń, Poland

e-mail: \{gruszka,pietrusz\}@umk.pl 